\title{
Hypolipidemic activity of Coriandrum sativum in diabetic dyslipidemic rats
}

\author{
Sayan Das, Vikram Rajadnya*, Revati Kothari, A. V. Tilak, Siddhi Raveendran, \\ Teja Deshpande
}

Department of Pharmacology, Dr. DY Patil Medical College and Hospital, Pune,

Maharashtra, India

Received: 11 April 2019

Accepted: 07 May 2019

*Correspondence to:

Dr. Vikram Rajadnya,

Email: med.pharma@dpu.edu.in

Copyright: (C) the author(s), publisher and licensee Medip Academy. This is an openaccess article distributed under the terms of the Creative Commons Attribution NonCommercial License, which permits unrestricted noncommercial use, distribution, and reproduction in any medium, provided the original work is properly cited.

\begin{abstract}
Background: Metabolic syndrome is described as the clustering of obesity, aberrant glucose metabolism, dyslipidemia and hypertension. A characteristic pattern, termed diabetic dyslipidemia, consists of low HDL, increased triglycerides and postprandial lipemia. This pattern is most frequently seen in type 2 diabetes and may be a treatable risk factor for subsequent cardiovascular disease. This study was designed to compare the hypolipidemic activity of Coriandrum sativum $L$. with the standard antidiabetic drug, metformin in streptozotocin induced diabetic rats.

Methods: Streptozotocin (STZ) was used to induce diabetes in the rats. The hypolipidemic activity of Coriandrum sativum seed extract was compared to the standard drug metformin. 4 groups $(n=8)$ (normal control, diabetic control, streptozotocin+Coriandrum sativum and streptozotocin+metformin). The drugs were administered once daily for 28 days following which lipid profile was estimated on $28^{\text {th }}$ day by using blood sample collected from the retro-orbital space.

Results: STZ induced diabetes and also lead to dyslipidemia. Oral administration of CS seed extracts significantly lowered total cholesterol (TC), LDL:HDL ratio, TC:HDL ratio, thus, reducing the cardiovascular risk. HDL levels were slightly increased with CS seed extract compared to diabetic control group but not statistically significant. There was also statistically insignificant reduction in the atherogenic index with CS seed extract compared to diabetic control.

Conclusions: CS seed extract $(40 \mathrm{mg} / \mathrm{kg})$ orally may have considerable therapeutic benefit as a hypolipidemic agent and can be suggested as a potential dietary add on.
\end{abstract}

Keywords: Atherogenic index, Cardiovascular risk, Coriandrum sativum, Dyslipidemia, HDL, Metabolic syndrome, Metformin

\section{INTRODUCTION}

Metabolic syndrome is described as the clustering of obesity, aberrant glucose metabolism, dyslipidemia and hypertension. Metabolic syndrome is also called as insulin resistance syndrome. ${ }^{1}$ Physical inactivity and obesity can lead to insulin resistance in genetically predisposed individuals. Among the metabolic abnormalities that commonly accompany diabetes are disturbances in the production and clearance of plasma lipoproteins.
Moreover, development of dyslipidemia may be a harbinger of future diabetes. A characteristic pattern, termed diabetic dyslipidemia, consists of low high-density lipoprotein (HDL), increased triglycerides (TG), and postprandial lipemia. This pattern is most frequently seen in type 2 diabetes and may be a treatable risk factor for subsequent cardiovascular disease. Dyslipidemia is one of the major risk factors for cardiovascular disease in diabetes mellitus. The characteristic features of diabetic dyslipidemia are a high plasma triglyceride concentration, 
low HDL cholesterol concentration and increased concentration of small dense LDL-cholesterol particles. The lipid changes associated with diabetes mellitus are attributed to increased free fatty acid flux secondary to insulin resistance.

Persons with metabolic syndrome are estimated to have twice the risk of developing CHD and five times the risk of developing diabetes than those without the syndrome. In addition, they have an increased cardiovascular mortality. ${ }^{2}$

Although many drugs are available to treat underlying clinical disorders, long term use of these agents are associated with many adverse drug reactions and disease control is not satisfactory in the long run.

Another approach is the use of alternative medicine such as herbal medicine, which provides significant clinical benefits in the correction of underlying abnormalities of metabolic syndrome. Main advantages of herbal medicines are low cost and minimal side effects.

Coriandrum sativum Linn. (Apiaceae), commonly known as coriander, cilantro, and dhania is an annual herb commonly used in ayurvedic medicine and also in cuisine in Middle East, Latin America and Asia. ${ }^{3}$ Pharmacological studies have demonstrated possible hypoglycemic, hypolipidemic, antihypertensive, anti-inflammatory, anxiolytic, antimicrobial, diuretic and cognition improvement activities in experimental animals. ${ }^{4,5}$

The aim ans objective of this study was designed to compare the hypolipidemic effect of Coriandrum sativum $L$. with the standard antidiabetic drug, metformin in streptozotocin induced diabetic rats. With the following objectives to study the hypolipidemic activity of Coriandrum sativum and to compare the hypolipidemic activity of Coriandrum sativum with metformin.

\section{METHODS}

Institutional Animal Ethics Committee (IAEC) clearance was obtained before start of the study.

Total 32 experimentally inexperienced Sprague-Dawley rats of Rattus norvegicus species (either sex weighing 100$200 \mathrm{~g}$ ) were acquired from the animal house of Dr. D.Y. Patil Medical College, Pimpri, Pune, Maharashtra, India and were fed with commercially available 'Nutrimix Std1020 'and water ad libitum.

Aqueous extract of Coriandrum sativum seeds was used for this study. Both the drugs, CS (40 mg/kg) and metformin $(90 \mathrm{mg} / \mathrm{kg}$ ), were administered orally once daily for 28 days. ${ }^{4,6}$ Streptozotocin (STZ) (40 mg/kg) intraperitoneally was used to induce diabetes in the rats. ${ }^{7}$ The lipid levels were estimated on $28^{\text {th }}$ day by using blood sample collected from the retro-orbital space.
The rats were divided into following groups $(n=8$ in each group).

Table 1: Groups of rats.

\begin{tabular}{|lll|}
\hline Group & Type & Dose \\
\hline Group 1 & Normal control & $\begin{array}{l}\text { Distilled water (p.o.) } \\
10 \mathrm{ml} / \mathrm{kg}\end{array}$ \\
\hline Group 2 & $\begin{array}{l}\text { Diabetic } \\
\text { control }\end{array}$ & $\begin{array}{l}\text { Streptozotocin (I.P.) } \\
40 \mathrm{mg} / \mathrm{kg}\end{array}$ \\
\hline \multirow{3}{*}{ Group 3 } & $\begin{array}{l}\text { Streptozotocin+ } \\
\text { Coriandrum } \\
\text { sativum }\end{array}$ & $\begin{array}{l}\text { Streptozotocin (I.P.) } 40 \\
\mathrm{mg} / \mathrm{kg}+\text { Coriandrum } \\
\text { sativum (P.O.) } 40 \mathrm{mg} / \mathrm{kg}\end{array}$ \\
\hline & $\begin{array}{l}\text { Streptozotocin } \\
\text { + metformin }\end{array}$ & $\begin{array}{l}\text { Streptozotocin (I.P.) 40 } \\
\mathrm{mg} / \mathrm{kg}+\text { Metformin (P.O.) } \\
\text { Group } \mathrm{mg} / \mathrm{kg}\end{array}$ \\
\hline
\end{tabular}

\section{Monitoring lipid profile}

The lipid levels were estimated on $28^{\text {th }}$ day using blood sample collected from the retro-orbital space.

- Total cholesterol,

- Triglyceride,

- HDL (High Density Lipoprotein),

- LDL ( Low Density Lipoprotein)

- LDL will be estimated by Friedewald's equation.

\section{LDL=Total Cholesterol-HDL-TG/5.}

The data was compiled and analysed by using the statistical package, Primer of Biostatistics 7.0. Results are expressed as mean $\pm \mathrm{SD}$ and statistical significance between means was analyzed using one-way analysis of variance (ANOVA) followed by Tukey's multiple comparison test. $\mathrm{P}$ value $<0.05$ was considered as statistically significant.

\section{RESULTS}

As per the methods, the study was carried out in 4 different groups. The results are expressed as mean \pm SD.

\section{Effect on total cholesterol (TC)}

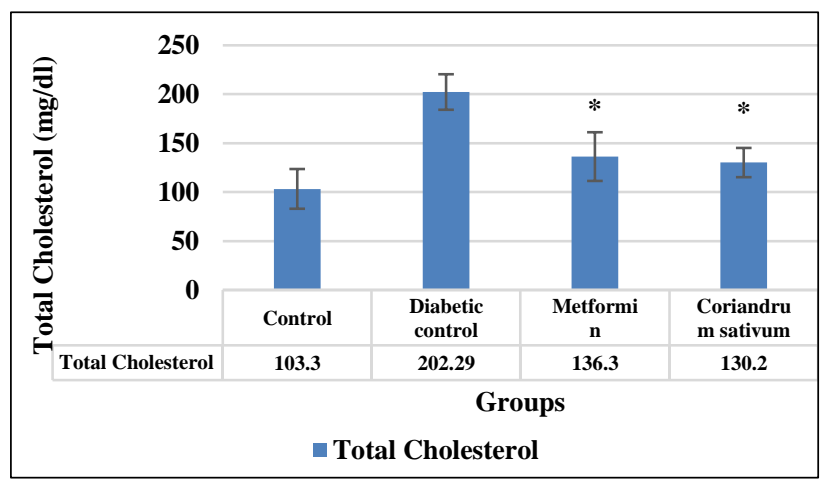

Figure 1: Effect on mean total cholesterol (TC) levels on 28th day. 
The mean total cholesterol levels on $28^{\text {th }}$ day of control group had significant statistical difference with diabetic control, showing that there was increased mean total cholesterol levels among the diabetic rats. CS $(40 \mathrm{mg} / \mathrm{kg})$ and metformin significantly lowered the mean total cholesterol level compared to group 2. However, the mean cholesterol levels of group 3 and 4 were comparable.

\section{Effect on triglyceride (TG)}

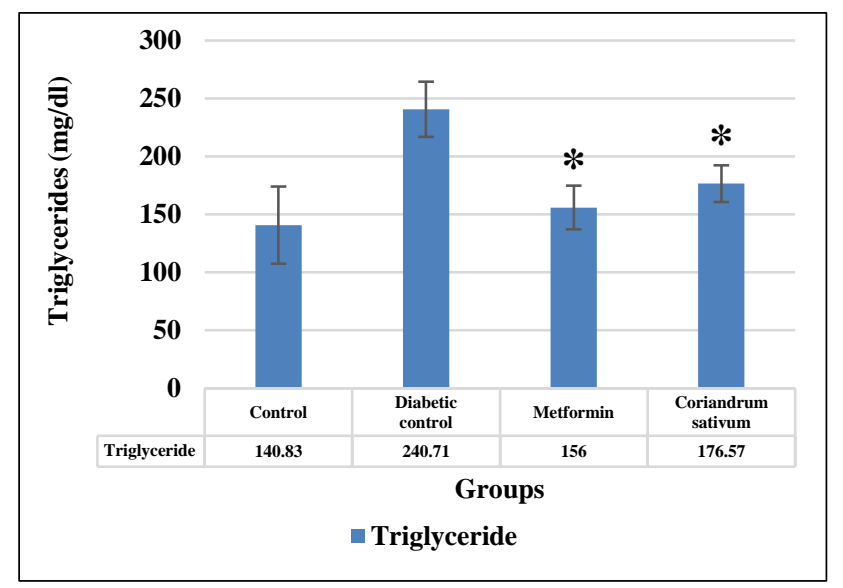

Figure 2: Effect on mean triglyceride (TG) levels on day $28^{\text {th }}$.

The mean triglyceride levels on $28^{\text {th }}$ day of group 1 had significant statistical difference with group 2, showing that there was increased mean triglyceride levels among the diabetic rats. CS $(40 \mathrm{mg} / \mathrm{kg})$ and metformin significantly lowered the mean triglyceride level as compared to group 2 . However, the reduction of triglyceride levels of group 3 and 4 were comparable.

\section{Effect on High-Density Lipoproteins (HDL)}

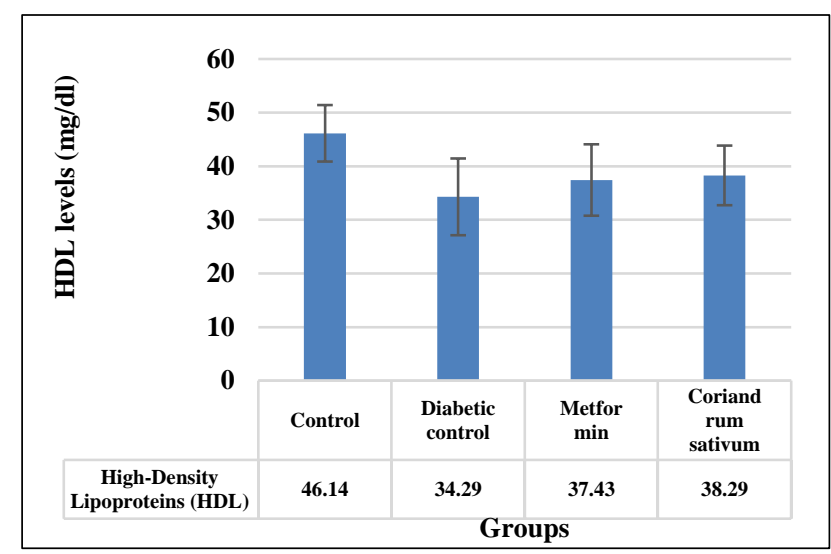

Figure 3: Effect on mean High-Density Lipoproteins (HDL) levels on $28^{\text {th }}$ day.

Metformin increased the mean High-Density Lipoproteins (HDL) levels and was comparable to group 2. The increase in mean HDL levels was comparable in group 3 and 4.

\section{Effect on Low-Density Lipoproteins (LDL)}

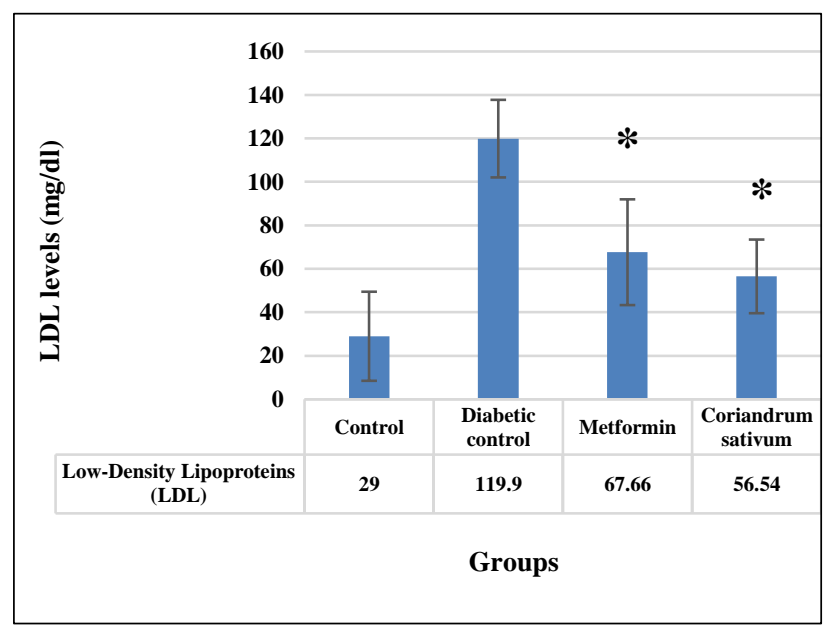

Figure 4: Effect on mean Low-Density Lipoproteins (LDL) levels on $28^{\text {th }}$ day.

CS $(40 \mathrm{mg} / \mathrm{kg}$ ) and metformin significantly reduced the mean Low-Density Lipoproteins (LDL) levels as compared to group 2. However, the mean LDL levels of group 4 were lower compared to group, however the difference wasn't statistically significant.

\section{Effect on Atherogenic Index (AI)}

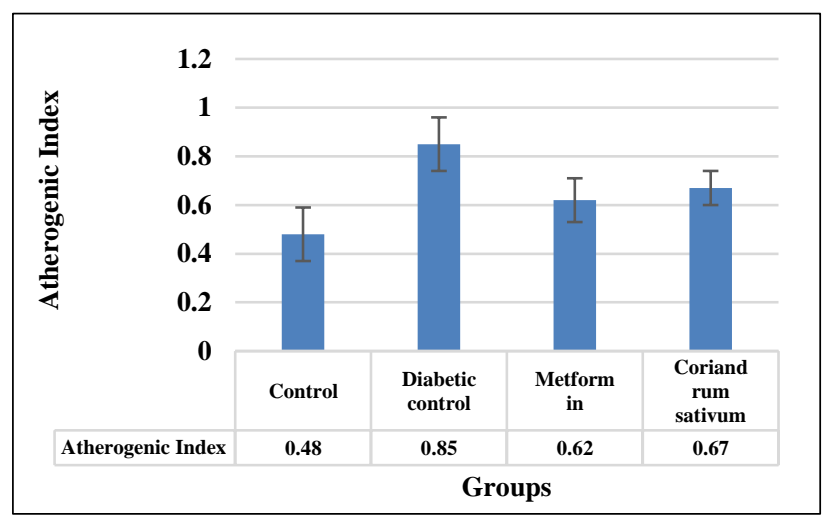

Figure 5: Effect on mean atherogenic index on $28^{\text {th }}$ day.

The mean atherogenic index in both groups 3 and 4 was comparable to group 2. Also, the mean atherogenic index obtained with group 4 was comparable to group 3 . There was no significant difference between all the groups.

\section{Effect on LDL:HDL ratio}

The mean Low-Density Lipoproteins: High-Density Lipoproteins (LDL:HDL) ratio of group 4 was comparable to group 3. Thus, it showed that the reduction in the cardiovascular risk with CS (40 mg/kg) was comparable to that of metformin. 


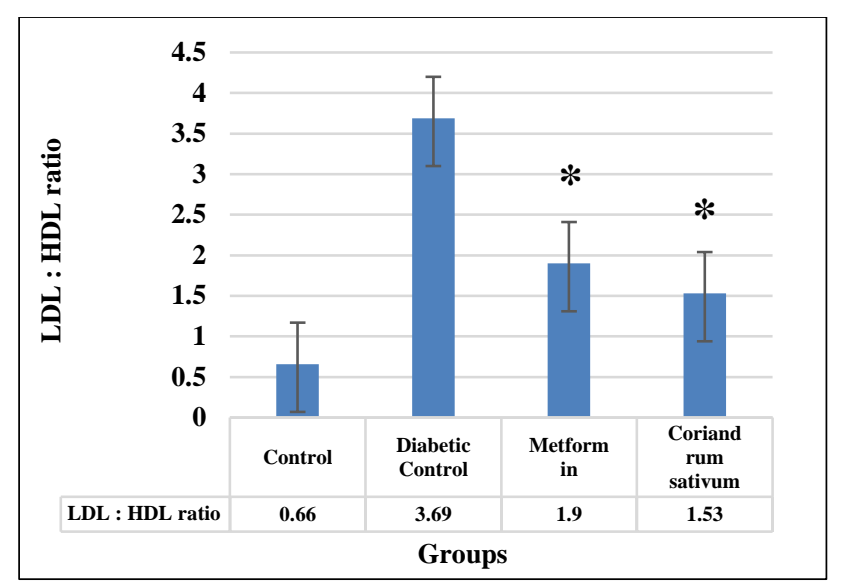

Figure 6: Effect on mean LDL:HDL ratio on day $28^{\text {th }}$.

\section{Effect on TC:HDL ratio}

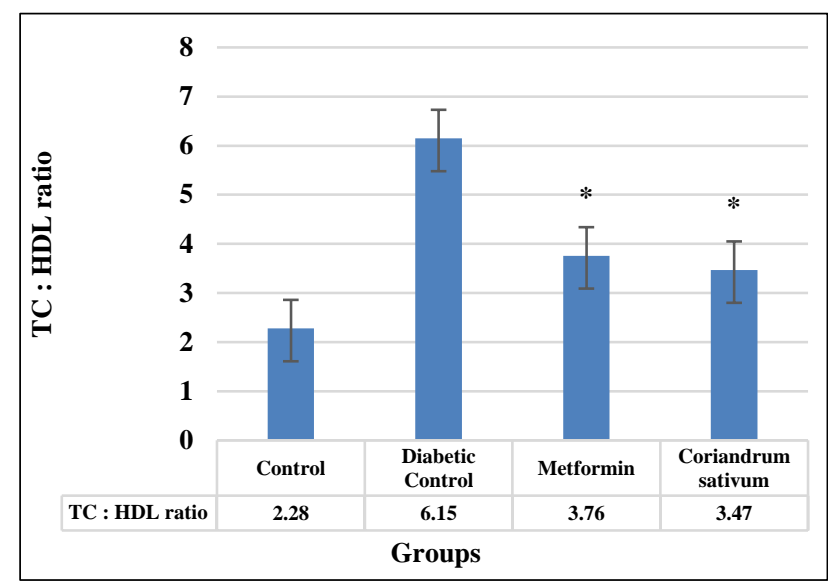

Figure 7: Effect on mean TC: HDL ratio on $28^{\text {th }}$ day.

CS (40 mg/kg) and metformin significantly reduced the mean total cholesterol: high-density lipoproteins (TC: HDL) ratio as compared to group 2. However, the mean TC: HDL ratio of group 4 was comparable to group 3. Thus, it showed that Coriandrum sativum seed extract significantly reduced risk of atherogenicity.

\section{DISCUSSION}

Persons with metabolic syndrome are estimated to have twice the risk of developing CHD and five times the risk of developing diabetes than those without the syndrome. In addition, they have an increased cardiovascular mortality. ${ }^{8}$ Lifestyle changes are the cornerstone of treatment. These lifestyle changes include diet low in saturated fat and cholesterol, weight reduction, increased physical activity and stress management. ${ }^{9}$

Although many drugs are available to treat diabetic dyslipidemia, long term use of these agents are associated with many adverse drug reactions and disease control is not satisfactory in the long run. Another approach is the use of alternative medicine such as herbal medicine, which provides significant clinical benefits in the correction of underlying abnormalities of metabolic syndrome.

Coriandrum sativum seed extract significantly lowered total cholesterol, triglyceride and LDL levels than control group ( $\mathrm{p}<0.05$ ). This lipid lowering effect produced by CS (40 mg/kg) was comparable to metformin $(90 \mathrm{mg} / \mathrm{kg})$. HDL levels were unexpectedly increased with CS in comparison to diabetic control group, but the difference was not statistically significant. There was a reduction in mean atherogenic index in the CS treated diabetic rats compared to group 2 but the difference was not statistically significant. However, LDL:HDL ratio and TC:HDL ratio was significantly reduced compared to group 2 whereas the reduction was comparable to metformin.

Present study findings are in agreement with other studies from literature, like Sreelatha $S$ et al, reported that both Coriandrum sativum leaf and stem extract significantly decreased the levels of cholesterol, triglycerides, LDL and VLDL. ${ }^{5}$

Aissaoui A et al, had also demonstrated that there was an increase in atherogenic index, LDL:HDL ratio and TC:HDL ratio in normal Meriones shawi rats but a decrease in the values in obese-hyperglycemichyperlipidemic $(\mathrm{OHH})$ Meriones shawi rats. ${ }^{4}$ Naquvi KJ et al, demonstrated that Coriandrum sativum fruits had significant reduction of total cholesterol levels and significant rise in HDL levels in streptozotocin induced diabetic rats. ${ }^{10}$

A study published by Das et al, has demonstrated Coriandrum sativum seed extract to have antihyperglycemic action in streptozotocin induced diabetic rats. ${ }^{11}$

Rajeshwari $U$ et al, conducted a clinical study where the results with Coriandrum sativum seed extract showed significant reduction of total cholesterol, triglycerides, LDL, and VLDL levels along with a rise in HDL levels. ${ }^{12}$ Dhanapakiam $\mathrm{P}$ et al, had also demonstrated a significant hypolipidemic action. ${ }^{13}$

Both Coriandrum sativum leaves and seeds are safe to use. Due to the long history of safe ingestion of fresh leaves and the dried seeds in most of the traditional cooking, this can be considered as a safe alternative. Coriandrum sativum seed extract as a hypolipidemic agent can be used as an add on drug or as a diet supplement. Mechanism of hypolipidemic action of Coriandrum sativum seed extract is not clearly understood.

The exact chemical constituent responsible for the observed hypolipidemic remains speculative.

Thus, Coriandrum sativum seed extract can target many arms of metabolic syndrome such as hyperglycemia and dyslipidemia. 


\section{CONCLUSION}

Oral administration of Coriandrum sativum seed extract at a dose of $40 \mathrm{mg} / \mathrm{kg}$ has shown hypolipidemic activity in streptozotocin induced diabetic dyslipidemic rats suggesting that Coriandrum sativum may have considerable therapeutic benefit as a hypolipidemic agent and can be suggested as a potential dietary add on.

\section{Funding: No funding sources}

Conflict of interest: None declared

Ethical approval: The study was approved by the Institutional Animal Ethics Committee

\section{REFERENCES}

1. Petrucelli OM. The metabolic syndrome. Northeast Florida Med. 2008;59(3):18-21.

2. Malik S, Wong ND, Franklin SS, Kamat TV, L Ítalien GJ, Pio JR, et al. Impact of the metabolic syndrome on the mortality from coronary heart disease, cardiovascular disease, and all causes in United States adults. Circulation. 2004;110(10):1245-50.

3. Sahib NG, Anwar F, Gilani AH, Hamid AA, Saari N, Alkharfy KM. Coriander (Coriandrum sativum L.): a potential source of high-value components for functional foods and neutraceuticals: a review. Phytother Res. 2013;27:1439-56.

4. Aissaoui A, Zizi S, Israili ZH, Lyoussi B. Hypoglycemic and hypolipidemic effects of Coriandrum sativum L. in Meriones shawi rats. J Ethnopharmacol. 2011;137(1):652-61.

5. Sreelatha S, Inbavalli R. Antioxidant, antihyperglycemic, and antihyperlipidemic effects of Coriandrum sativum leaf and stem in alloxan-induced diabetic rats. J Food Sci. 2012;77:119-23.

6. Pirkola J, Tammelin T, Bloigu A, Pouta A, Laitinen J, Ruokonen A, et al. Prevalence of metabolic syndrome at age 16 using international diabetes federation pediatric definition. Arch Dis Child. 2008;93:945-5.
7. Akbarzadeh A, Norouzian D, Mehrabi MR, Jamshidi S, Farhangi A, Verdi AA, et al. Induction of diabetes by Streptozotocin in Rats. Indian $\mathrm{J}$ Clin Biochem. 2007;22(2):60-4.

8. Malik S, Wong ND, Franklin SS, Kamat TV, L Ítalien GJ, Pio JR, et al. Impact of the metabolic syndrome on the mortality from coronary heart disease, cardiovascular disease, and all causes in United States adults. Circulation. 2004;110(10):1245-50.

9. Grundy SM, Brewer HB, CLeeman JI, Smith SC, Lenfant C. Definition of metabolic syndrome: report of the national heart, lung, and blood institute/American Heart Association conference on scientific issues related to definition. Circulation. 2004;109:433-8.

10. Naquvi KJ, Ali M, Ahamad J. Antidiabetic activity of aqueous extract of coriandrum sativum fruits in streptozotocin induced rats. Int J Pharm Pharmaceut Sci. 2012;4(1):239-41.

11. Das S, Chaware S, Narkar N, Tilak AV, Raveendran S, Rane P. Antidiabetic activity of Coriandrum sativum in Streptozotocin induced diabetic rats. Int $\mathbf{J}$ Basic Clin Pharmacol. 2019;8(5):925-9.

12. Rajeshwari U, Iyer S, Bondada A. Comparison of aniseeds and coriander seeds for antidiabetic, hypolipidemic and antioxidant activities. Spatula DD. 2011;1(1):9-16.

13. Dhanapakiam P, Joseph JM, Ramaswamy VK, Moorthi M, Kumar AS. The cholesterol lowering property of coriander seeds (Coriandrum sativum): mechanism of action. J Environ Biol. 2008;29(1):536.

Cite this article as: Das S, Rajadnya V, Kothari R, Tilak AV, Raveendran S, Deshpande T.

Hypolipidemic activity of Coriandrum sativum in diabetic dyslipidemic rats. Int J Basic Clin Pharmacol 2019;8:1393-7. 